\title{
Autonomie und soziale Sicherheit - Anforderungen an arbeits- und sozialrechtliche Regulierung
}

\begin{abstract}
Arbeitsrecht und Sozialrecht bestimmen mit, wie Menschen ihre Arbeits- und Lebensbedingungen selbst bestimmen und gestalten können. Die Regelungsbedürfnisse ändern sich aber: Das „Normalarbeitsverhältnis“ langjähriger ununterbrochener Vollzeittätigkeit reicht jedenfalls als Leitbild arbeits- und sozialrechtlicher Regulierung nicht mehr aus. Die Anforderungen der Erwerbsarbeit kollidieren immer häufiger und für immer mehr Menschen mit anderen Lebenssituationen und gesellschaftlichen Einbindungen, wozu insbesondere Familie, Qualifizierung, gesundheitliche Veränderungen gehören. Für solche sozialen Situationen sollte das Recht garantierte Optionen und sozialen Schutz anbieten - was es vereinzelt bereits auch tut.
\end{abstract}

\section{"Abschied vom Normal- arbeitsverhältnis" auch im Arbeits- und Sozialrecht?}

Veränderungen der Arbeitswelt und des Arbeitsmarktes haben dazu geführt, dass die typischen Lebensläufe erwerbstätiger Menschen sich geändert und differenziert haben. Politik und Gewerkschaften, Arbeits- und Industriesoziologie haben sich in den letzten Jahrzehnten aus unterschiedlichen Perspektiven mit den Veränderungen in der Organisation der Arbeit beschäftigt, die zusammengefasst als „Postfordismus“ bezeichnet werden. Gängige Versuche der begrifflichen Erfassung beziehen sich auf „Flexibilität“ (Sennett 2000), „Subjektivierung“, „Entgrenzung“, „Krise der Repräsentation“, „Prekarität/ Prekarisierung" (Brinkmann et al. 2006), „Arbeitskraftunternehmertum“ (Voß/ Pongratz 1998; Welti 2001a).

In diesem Kontext wird insbesondere das „Normalarbeitsverhältnis“ langjähriger ununterbrochener Vollzeittätigkeit als Leitbild der Regulierung infrage gestellt. Dieses Leitbild geht davon aus, dass ein Mensch sein Leben überwiegend der Erwerbsarbeit zur Verfügung stellt: Statt die Erwerbsarbeit ins Leben der Menschen einzubeziehen, wird der Mensch in den fremdbestimmten Rhythmus der Erwerbsarbeit integriert. Die familiäre, kulturelle und bürgerschaftliche Dimension des Lebenslaufs und der Gesellschaft wird damit an den Rand gedrängt.
Eine neue Perspektive ist vonnöten, die im Sinne eines Konzepts gesellschaftlicher Inklusion die gesellschaftlichen Voraussetzungen und Einbettungen der Arbeitskraft und ihrer Reproduktion wie Familien- und Sorgearbeit, Bildung und Qualifikation systematischer berücksichtigt und diesem Erfordernis auch in den Strukturen des Arbeits- und Sozialrechts Rechnung trägt. Gesellschaftlicher Wandel, zunehmend diskontinuierliche Erwerbsbiografien und Individualisierung brauchen nicht weniger, sondern mehr und anderen durchsetzbaren rechtlichen Schutz.

Abschnitt 2 skizziert im Überblick die einschlägigen sozial- und rechtswissenschaftlichen Diskurse, die dieses Problem erkannt und erste Ansätze für entsprechende neue Regulierungsanforderungen erarbeitet haben. Zugleich werden deren bisherige Begrenzungen bzw. notwendige Perspektivenerweiterungen aufgezeigt. Dass der als notwendig beschriebene Regulierungsbedarf zumindest punktuell unter Rückgriff auf vorhandene arbeits- und sozialrechtliche Instrumente bearbeitet werden kann, zeigt Abschnitt 3. Er macht jedoch auch deutlich, dass die bestehenden rechtlichen Optionen noch zu kurz greifen und aus unterschiedlichen Gründen das Ziel, Autonomie ohne Prekarität zu schaffen, nicht erreichen können, was auf weiteren Diskussions- und Forschungsbedarf wie auch auf die im Abschnitt 4 angesprochenen Probleme fehlender Rechtsdurchsetzung verweist.

\section{Anknüpfungspunkte im sozial- und rechtswissen- schaftlichen Diskurs}

\subsection{LEBENSLAUFPERSPEKTIVE UND DIE PROBLEMATIK DER ÜBERGÄNGE}

Aus feministischer Perspektive werden die mit dem Leitbild des Normalarbeitsverhältnisses verbundenen Probleme schon lange diskutiert. Das bisherige Leitbild reproduziere das Modell des männlichen „Familienernährers“ (Ostner 1995) und trage zu einer fremdbestimmten Arbeitsteilung und einem asymmetrischen Geschlechterverhältnis bei (Nickel 1999). Die Notwendigkeit einer geschlechterdemokratischen Perspektive (Stolz-Willig 2001) ergebe sich auch aus dem Wandel der Geschlechterverhältnisse und Familienstrukturen (Klenner/Pfahl 2008). Es könne

Eva Kocher, Dr. jur., Professorin an der Juristischen Fakultät der Europa-Universität Frankfurt/Oder. Arbeitsschwerpunkte: Bürgerliches Recht, Europäisches und Deutsches Arbeitsrecht sowie Zivilverfahrensrecht. e-mail: kocher@euv-frankfurt-o.de Felix Welti, Dr. jur., Professor am Fachbereich Gesundheit, Pflege, Management der Hochschule Neubrandenburg, Arbeitsschwerpunkte: Sozialrecht und Verwaltungsrecht. e-mail: mail@felix-welti.de 
heute nicht mehr unproblematisch davon ausgegangen werden, dass das gesellschaftliche Leben jenseits der Erwerbsarbeit, insbesondere private Fürsorge, von Frauen in unbezahlter Arbeit gestaltet werde. Damit entstünden neue Anforderungen an den Sozialstaat. Die vormals unentgeltlich von Ehefrauen, Müttern und (Schwieger-) Töchtern geleistete Fürsorge (Care) und Familienarbeit müsse neu verteilt werden (Bogedan 2008).

Erwerbsarbeit muss aber künftig generell nicht nur mit Kindererziehung und Pflege vereinbart werden können, sondern auch mit anderen gesellschaftlichen Einbindungen. Da immer mehr Menschen erwerbstätig sind, sein wollen oder sollen (vgl. $\$ 10$ SGB II), lässt sich die Erwerbsarbeit immer weniger scharf von der gesellschaftlichen Einbindung außerhalb der Erwerbsarbeit trennen. So beschränken sich Zeiten der Qualifizierung vielfach nicht mehr nur auf den Beginn des Erwerbslebens, denn der Anteil qualifizierter Arbeit steigt und der technische Fortschritt hat sich beschleunigt. Krankheit, Behinderung oder altersbedingte Leistungsminderungen treten - nicht zuletzt wegen einer länger werdenden Lebensarbeitszeit und höheren Leistungsanforderungen - auch zunehmend bei im Erwerbsleben stehenden Menschen auf. Ökonomisch gesehen geht es darum, dass die gesellschaftliche Arbeitskraft nicht nur genutzt, sondern auch reproduziert wird; politisch geht es um eine Gesellschaft, in der die Arbeitenden auch Zeit haben, die Früchte ihrer Anstrengungen zu genießen.

Der in der Arbeitsmarktforschung geprägte Begriff des „Übergangs“ (Schmid 2002, S. 233) erscheint geeignet, um solche Änderungen in der Organisation der Erwerbsarbeit an exemplarischen Problemen des Arbeits- und Sozialrechts zu diskutieren. Wenn Erwerbsarbeit immer häufiger und für immer mehr Menschen mit anderen Lebenssituationen und gesellschaftlichen Einbindungen zusammentrifft, muss politische und rechtliche Regulierung die Erwerbsarbeit in einer Lebenslaufsperspektive betrachten. Im Verlauf des Lebens setzen Menschen unterschiedliche Schwerpunkte, die sich zeitlich überlappen und zwischen denen Wechselverhältnisse und Übergänge bestehen. Der Begriff des Übergangs ist insofern zu erweitern um die Perspektive der Gleichzeitigkeit „gemischter Existenzen“" (Döring 2009).

\subsection{DIE FLEXICURITY-DEBATTE}

Die europäische Regelungsebene hat durch Öffnungen zwischen nationalen Arbeitsmärkten sowie Beschäftigungspolitiken einiges zur „Flexibilisierung“ beigetragen. Als eine Reaktion auf die unzureichende Regulierung auf europäischer Ebene sowie als Versuch, ein rechtspolitisch konsistentes Konzept für europäisierte Arbeitsmärkte zu entwickeln, wurde und wird auf europäischer Ebene insbesondere unter dem Stichwort „Flexicurity“ über neue Regulierungskonzepte des „Abschieds vom Normalarbeitsverhältnis“ diskutiert.

Der Begriff knüpft an Regulierungsmuster in den Niederlanden und in Dänemark an, die beanspruchen, in neuartiger Weise Flexibilität mit sozialer Sicherheit $\mathrm{zu}$ verbinden, um interne und externe Übergänge im Arbeitsleben zu erleichtern und umfassenden Sozialschutz zu gewährleisten (Klammer 2005; Blanke/ Bleses 2005; P. Hanau 2005). Doch greift diese bisherige Debatte um "Flexicurity“ zu kurz: Sie verengte sich im Laufe der Zeit auf die Gewährleistung unternehmerischer Flexibilität. Fragen des sozialen Schutzes wurden vernachlässigt. Zwischen den europäischen Akteuren sind die Inhalte eines „Flexicurity“-Konzepts zwar im Einzelnen umstritten. Mit dem Grünbuch „Ein modernes Arbeitsrecht für die Herausforderungen des 21. Jahrhunderts“ (Europäische Kommission 2006) hat die Europäische Kommission aber Pflöcke eingeschlagen, die "Flexicurity" heute überwiegend als Infragestellung bisheriger Ordnungsmuster ohne soziale Perspektive erscheinen lassen. Dem entsprechend kritisierte z. B. das Europäische Parlament zu Recht die Ausrichtung auf einen Abbau von Kündigungsschutz (vgl. BundesratDrs. 950/07).

Auch erscheint der Begriff der „Flexicurity" problematisch. Er suggeriert vielfach eine defensive und verkürzte Vorstellung von sozialer Sicherheit, die auf die Flexibilisierungswünsche der Unternehmen fokussiert und diese in gewissem Umfang sozial abzufedern versucht (vgl. Keller/Seifert 2008, S. 6ff.). Damit erscheint aber zumindest unausgesprochen das „Normalarbeitsverhältnis“ als einziges Leitbild des sozialen Schutzes, das unternehmerischen Flexibilisierungsinteressen entgegengesetzt werden müsse. Für Übergangssituationen in einer Lebenslaufperspektive muss in Abgrenzung dazu aber auch die Autonomie der Beschäftigten zu ihrem Recht kommen, deren Realisierung Flexibilität vom Arbeitgeber fordert.

\subsection{SOZIALES RECHT DER ARBEIT: AUTONOMIE UND SOZIALE SICHERHEIT}

Autonomie, Handlungsspielräume und Optionen der Beschäftigten, ihre Wahlfreiheit zwischen unterschiedlichen Lebensmodellen jenseits von Rollenzuschreibungen und Zwängen des (Arbeits-)Marktes sind keine neuen Themen. Schon Hugo Sinzheimer, Gustav Radbruch und Hermann Heller haben in der Weimarer Republik dem Begriff des sozialen Rechtsstaats einen Inhalt gegeben, der durch die Macht der Tarifparteien und des demokratischen Staates mehr Freiheit in der Arbeitswelt realisieren sollte. In heutigen Begriffen formuliert: Ein Soziales Recht der Arbeit verlangt nach „Autonomie ohne Prekarität“, nach „Flexibilität durch Sicherheit" und nach "guter Arbeit“ - und zwar für alle Beschäftigten in gleicher Weise, unabhängig von Qualifikation, Geschlecht und beruflicher Stellung.

Damit erhalten Fragen des sozialen Schutzes zwar eine zentrale, aber nicht eine ausschließliche Bedeutung. Die zunehmenden Anforderungen, das eigene Leben und die eigenen Arbeitskraft zu managen, werden heute ebenso als subjektiver Autonomiegewinn und als Aufstiegschance wie als belastende Überforderung, Kontrollverlust und Gefahr sozialen Abstiegs erlebt. Und mehr noch: Prekaritätserfahrungen sind nicht notwendig mit einer bestimmten sozialen Gestaltung oder Art und Weise der Beschäftigung verbunden. Wieweit eine Beschäftigungs- oder Übergangssituation als prekär erscheint und unter welchen Voraussetzungen Übergänge den Interessen der Unternehmen oder denen der Beschäftigten entsprechen und "gelingen“ können, ist abhängig von der rechtlichen Gestaltung und ihrer Wahrnehmung. Die rechtliche Regulierung kann und muss gewährleisten, dass Beschäftigte über garantierte Optionen und Handlungsmöglichkeiten verfügen, um Übergänge herzustellen und angemessen so zu gestalten, dass Lebensstandard, Arbeitsbedingungen und Risikosicherung so wenig wie möglich durch Rechtsverluste bedroht sind (Klammer 2005). 


\subsection{DER SUPIOT-REPORT}

Für das an punktuellen Situationen und festem Status orientierte Arbeits- und Sozialrecht ist mit der systematischen Bewältigung einer solchen Lebenslaufperspektive ein anspruchsvolles Programm formuliert. Bereits der "Supiot-Report“, der Forschungsbericht eines rechtsvergleichenden Teams, dessen Arbeit durch die EU-Kommission finanziert worden ist, beanspruchte, mögliche Regelungsinstrumente für den Wandel der Erwerbsarbeit zu entwickeln (Supiot 2001; Supiot et al. 1999). Normativ orientierte sich die Gruppe am Modell der „social citizenship“. Nach der Diagnose des Berichts liegt die Herausforderung vor allem darin, Regulierungen zum sozialen Schutz zu schaffen, die es ermöglichen, mit wachsender Unsicherheit umzugehen. Er kam zum Ergebnis, dass die auch hier benannten neuen - oder neu bewusst gewordenen - sozialen Risiken, Konstellationen und Interessenlagen im Arbeitsverhältnis nicht weniger, sondern anderer Regulierung bedürfen. Die Formen und Institutionen des Arbeits- und Sozialrechts wie Gesetz, Tarifvertrag und Sozialversicherung werden folglich nicht überflüssig, sondern müssen revitalisiert werden.

In der Öffentlichkeit erlangte vor allem die im Supiot-Bericht begründete These von der Notwendigkeit von „Ziehungsrechten " breitere Aufmerksamkeit (Mückenberger 2007). Mit diesem wenig gängigen Begriff werden Optionsrechte der Beschäftigten z.B. auf Teilzeitarbeit oder arbeitsfreie Zeiten bezeichnet, die ein erneuertes Arbeitsrecht prägen sollen.

Den rechtsvergleichenden Überlegungen des Supiot-Berichts fehlt es allerdings an konkreten Perspektiven für einzelne Rechtsordnungen wie die deutsche. Die Debatte zeigt aber auf, dass hier dringender Bedarf an neuen und pfadangepassten (d.h. auf das jeweilige nationale Recht und seine Praxis bezogenen) Konzepten besteht. Dabei ist zu berücksichtigen, dass die gesellschaftliche Diskussion der letzten Jahrzehnte in Gesetzgebung und Rechtsprechung durchaus Spuren hinterlassen hat, anhand derer möglicherweise zukunftsweisende Regulierungsformen aufgedeckt werden können. Zum Teil sind ,garantierte Optionen“ bzw. „Ziehungsrechte“ im geltenden Arbeits- und Sozialrecht bereits angelegt (wie z.B. Elternzeit oder Bildungsurlaub). Ob und wie Ziehungsrechte wirklich etwas qualitativ Neues im Arbeitsrecht sein und in einen kohärenten Rahmen gestellt werden könnten, ist bislang aber kaum diskutiert worden. Auch wäre anhand der Defizite bisheriger Normsetzung und Normdurchsetzung zu diskutieren, auf welchen rechtlichen und sozialen Voraussetzungen erfolgreiche Regulierung beruhen muss.

Im Folgenden wird für einige wenige Regelungsbereiche dargestellt, mit welchen Instrumenten das deutsche Recht bereits auf die dargestellten Herausforderungen reagiert und welche Probleme sich ergeben. Wir beschränken uns dabei exemplarisch und ohne Anspruch auf Vollständigkeit auf einige Bereiche, für die bereits neue Regelungsinstrumente entwickelt worden sind: Dazu zählen Fragen der Sozialversicherungspflicht (Abschnitt 3.1), der Arbeitszeit (Abschnitt 3.2 und 3.3) sowie die Integration gesundheitlicher Einschränkungen im Erwerbsleben (Abschnitt 3.4).

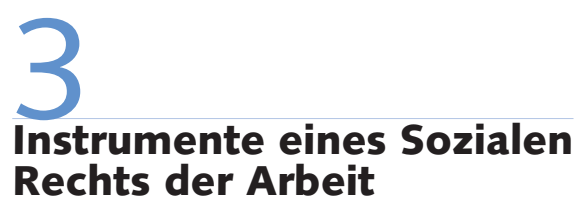

\subsection{SOZIALVERSICHERUNGSPFLICHT BEI WECHSELNDEN BESCHÄFTI- GUNGSFORMEN}

Innerhalb der Sozialversicherung ist das Problem des gleichzeitigen Zusammentreffens unterschiedlicher Risiken weitgehend dadurch gelöst, dass die Träger wechselseitig Beiträge leisten, wenn durch ein Risiko das Erwerbseinkommen ausfällt. Beispielsweise zahlt die Krankenkasse während des Krankengeldbezugs Arbeitslosenversicherungsbeiträge, und die Bundesagentur entrichtet während Arbeitslosigkeit Krankenversicherungsbeiträge. Dadurch wird der Versicherungsschutz aufrecht erhalten. Der Übergang zwischen unterschiedlichen Beschäftigungs- und Versicherungsformen wird aber meist problematisch, wenn keine Lohnersatzleistung (mehr) beansprucht werden kann. Erziehungszeiten werden in etlichen Bereichen der sozialen Sicherung zumindest berücksichtigt, etwa durch Kindererziehungszeiten bei der Rente und beim Anwartschaftserhalt in der Arbeitslosenversicherung (vgl. $\$ 56$ SGB VI; $\S 26$ Abs. 2 SGB III). Qualifikationszeiten oder längere Krankheit können aber oft zu Anspruchsverlusten führen. Auch gewährleistet das Sozialleistungssystem eher, dass Ansprüche dem Grunde nach erhalten bleiben, nicht aber der Höhe nach, sodass z.B. der Wechsel in Teilzeitarbeit den Umfang der Sicherung oft unter das Minimum drückt. Wenn man von Arbeit gerade leben kann, dann kann man es von der darauf berechneten Lohnersatzleistung nicht mehr. Auch hier wird deutlich, dass die bisherigen Regulierungsformen nur für einen Teil der Beschäftigten realisierbare Gestaltungsrechte bereithalten.

Das Zusammentreffen von anderen Übergangssituationen mit gesundheitlichen Problemen ist ebenfalls prekär. So ist das krankheitsbedingte Entgeltausfallrisiko von Selbstständigen in der gesetzlichen Krankenversicherung weiterhin nicht obligatorisch gesichert ( $\$ 44$ Abs. 2 Ziff. 2 SGB V; Biehl 2004). Krankheitsbedingte Erwerbsunterbrechungen können zum Verlust des Erwerbsminderungsrentenanspruchs führen ( $\$ 43$ SGB VI), weil der Anspruch auf Erwerbsminderungsrente in der gesetzlichen Rentenversicherung weiter an eine vorhergehende Pflichtversicherungszeit aufgrund versicherter Beschäftigung gebunden ist. Während Erziehungs- und Krankheitszeiten bei der Erwerbsminderungsrente zumindest anspruchsverlängernd berücksichtigt werden ( $\$ 43$ Abs. 4 SGB VI), ist dies für Studium oder Weiterbildung nicht der Fall.

Die Möglichkeit einer allgemeinen Versicherungspflicht ist speziell in der Kranken- und Pflegeversicherung überwiegend positiv bewertet worden. Es bleiben aber einerseits Probleme der Beitragslast, andererseits der Leistungsseite, etwa unter dem Aspekt des Entgeltrisikos. Nach dem Koalitionsvertrag der neuen Regierung ist eher eine weitere Entkoppelung von Krankenversicherung und Arbeitswelt zu befürchten, unter Aufgabe des Arbeitgeberbeitrags und möglicherweise auch des Krankengeldanspruchs.

Die Zuordnung zu einem Beschäftigungsstatus bleibt auch dann folgenreich, wenn schutzbedürftige Selbstständige in die Sozialversicherung einbezogen worden sind. Im Handwerk, in der Landwirtschaft und Fischerei sind Selbstständige schon seit Jahrzehnten pflichtversichert. Seit den 1970er Jahren sind weitere Öffnungen im Zugang zum Sozialversicherungsschutz für Nichterwerbstätige und Selbstständige vorgenommen worden (Bieback 1999; Welti 2001b). Die 1981 eingeführte Künstlersozialversicherung hat als einzige Reform dieser Art einen Mechanismus 
entwickelt, mit verfassungsrechtlicher Legitimation auch die Auftraggeber der Selbstständigen zu beteiligen (BVerfGE 75, 108), um so - im Verein mit Steuerzuschüssen - den Übergang in selbstständige Tätigkeit sozialrechtlich zu entlasten. Trotz dieser einzelnen Ansätze ist die soziale Sicherung der Selbstständigen in Deutschland im europäischen Vergleich insgesamt rückständig (Reinhard 2009).

Neben dem Zugang zur Sozialversicherung „dem Grunde nach“ sind auch die jeweiligen Zugangsbedingungen zu untersuchen. Im Bereich der Krankenversicherung scheint vor allem die Beitragsgestaltung freiwillig Versicherter ( $\$ 240$ SGB V) problematisch zu sein (dazu BVerfGE 103, 392; BSG, SozR 4-2500 \$224 Ziff. 1). Die Beiträge für die Versicherung von Selbstständigen und von Personen in atypischen Lebenslagen, wie z.B. Praktikanten oder Studierenden oberhalb des 30. Lebensjahres, sind im Verhältnis zum Einkommen sehr hoch. Die Rechtsprechung des Bundessozialgerichts hat zudem Beitragsbefreiung in Elternzeit und während des Bezugs von Krankengeld für freiwillig Versicherte nicht gelten lassen (BSG v. 26.5.2004, Az. B 12 P 6/03 R). Generell gilt: Das Problem des Übergangs zwischen Beschäftigungsformen lösen weder die aktuellen Zugangsbedingungen noch vorliegende Konzepte der Bürgerversicherung - oder nur um den Preis einer Aufgabe des Arbeitgeberbeitrags.

Letztlich deckt die rechtliche Regulierung nur einige soziale Risiken ab und vernachlässigt andere. Umbrüche im Lebenslauf treten aber meist nicht einzeln, sondern zusammen auf. Oft drohen dann in Übergangssituationen Rechtsverluste. Es kann zu Einbußen von Einkommen, Qualität der Arbeit, Aufstiegsmöglichkeiten, Arbeitsmarktpositionen und sozialer Sicherheit kommen. Insbesondere für Personen mit niedrigerem Arbeitseinkommen ist das Sicherungsniveau nicht ausreichend. Nach wie vor gilt, was bereits die gleichstellungsorientierte Sozialpolitikforschung aufgezeigt hat: Unterbrochene Erwerbskarrieren führen zu bedeutsamen Sicherungslücken.

\subsection{ARBEITSZEITKONTEN}

Im Arbeitsrecht ist die Arbeitszeitgestaltung ein zentrales Instrument der internen Flexibilität (Keller/Seifert 2007), um Wahlmöglichkeiten zur Vereinbarkeit von
Erwerbsarbeit mit anderen lebensphasenspezifischen Bedürfnissen sowie zum Gesundheitsschutz und der Verringerung von Zeitdruck und Stress zu ermöglichen (Brandl et al. 2008; Hildebrandt 2007; Klammer 2005). Formen der Arbeitszeitflexibilisierung von Gleitzeit über Teilzeitarbeit in ihren unterschiedlichen Ausprägungen bis hin zu Arbeitszeitkonten sind arbeitsrechtlich seit Langem geregelt; der Regelungsschwerpunkt liegt dabei auf tariflicher und betrieblicher Ebene. Was die soziale Sicherung bei flexibilisierter Arbeitszeit angeht, so konzentriert sich die Debatte auf die Regelung der Wertguthaben in Zeitkonten, ihre sozialversicherungsrechtliche Behandlung und Insolvenzsicherung. Der Sozialversicherungsschutz kann bereits nach dem Gesetz zur sozialrechtlichen Sicherung flexibler Arbeitszeitregelungen vom 6.4.1998 und nach dem zum 1.1.2009 in Kraft getretenen Gesetz zur Verbesserung der Rahmenbedingungen für die Sicherung flexibler Arbeitszeitregelungen an flexible Einsatzformen im Arbeitsverhältnis angepasst werden (\$\$ 7-7g SGB IV; Hanau/Veit 2009). Voraussetzung ist, dass zwischen Arbeitgeber und Beschäftigtem entsprechende Wertguthabenvereinbarungen getroffen wurden. Bislang sind diese Instrumente leider weder rechtssystematisch noch empirisch aufgearbeitet worden. Sie scheinen in größerem Stil nur für die Übergangssituation der Altersteilzeit verstärkt genutzt worden zu sein (Kerschbaumer 2009).

Woran dies liegt, ob die geltenden Regelungen bürokratisch, kostenintensiv und unattraktiv sind (Rolfs/Witschen 2009, S. 302) und ob solidarische Alternativen bestehen (Schietinger 2008), würde einen genaueren Blick lohnen, da es sich unter dem Gesichtspunkt der Lebenslaufperspektive um innovative und interessante Instrumente handelt. Hier besteht Forschungsbedarf. Zuletzt sind mithilfe tariflich und betrieblich flexibilisierter Arbeitszeit und mit dem sozialrechtlichen Instrumentarium der Kurzarbeit die konjunkturellen Krisenerscheinungen vor allem in der Metallindustrie abgefedert worden. Wieder einmal wurde deutlich, dass Arbeits- und Sozialrecht flexible Lösungen ermöglichen, die aber heute fast nur genutzt werden, wenn sie betrieblich veranlasst sind. Aus Sicht eines Sozialen Rechts der Arbeit wäre zu fragen, ob mit ähnlichem Instrumentarium auch individuelle Übergangssituationen bewältigt werden könnten.

\subsection{GARANTIERTE OPTIONEN AUF TEILZEIT}

Empirisch lässt sich feststellen, dass Langzeitkonten überwiegend von den höher qualifizierten Dienstleistungsbeschäftigten genutzt werden, während im gewerblichen und operativen Bereich das Interesse am geringsten ist. Gerade die Gruppen, die am dringendsten auf zeitliche Entlastung angewiesen sind, wie Beschäftigte mit Kindern oder Pflegeaufgaben, haben kaum Guthaben mit dem Langzeitkonto aufbauen können (Wotschack et al. 2008). In der mittleren Lebensphase hat offensichtlich vor allem die tägliche Balance von beruflichen und außerberuflichen Aktivitäten einen hohen Stellenwert. Dies ist nur einer der Gründe für die hohe Relevanz der Teilzeitarbeit.

Das deutsche Recht enthält bereits eine Reihe von Ansprüchen auf Reduzierung der Arbeitszeit im Teilzeit- und Befristungsgesetz, im Elternzeitrecht und im Pflegezeitgesetz $(\$ 8$ TzBfG, $\$ 15$ V-VII BEEG, PflegeZG; vgl. z.B. Waas 2000; Opitz 2005; Berger-Delhey 2009), also garantierte Optionen und Gestaltungsansprüche. $\$ 8$ TzBfG ist dabei ein im Grundsatz unkonditioniertes Recht auf Veränderung der Arbeitszeit, dem allerdings kein sozialrechtlicher Ausgleich des Einkommensverlusts entspricht.

Diese und ähnliche Optionen wurden im systematischen Kontext des Diskriminierungsschutzes diskutiert und entwickelt. Die Geschlechtergleichstellungsgesetze im öffentlichen Dienst können als Vorreiter in der Begründung von Gestaltungsansprüchen angesehen werden. Solche Gleichstellungs- und Gestaltungsansprüche stellen eine Weiterentwicklung von Gleichbehandlungs- oder Antidiskriminierungsansprüchen dar. Das US-amerikanische und das kanadische Antidiskriminierungsrecht kennen mit den Ansprüchen auf angemessene Vorkehrungen („reasonable accommodation“) bereits eine eigenständige Kategorie von Gestaltungsansprüchen, die in ihrer Reichweite zwischen dem Diskriminierungsverbot und den positiven Maßnahmen oder Förderpolitiken angesiedelt sind (Waddington/Hendriks 2002). Im europäischen und deutschen Recht werden angemessene Vorkehrungen noch nicht systematisch als eigenständiges Rechtsinstitut im Kontext des Diskriminierungsschutzes diskutiert. In der Gesetzgebung gibt es jedoch schon 
erste Ansätze in diese Richtung, vor allem gegen die Diskriminierung und für die Inklusion behinderter Menschen (vgl. die Behindertenrechtskonvention; Art. 5 RL 2000/78/EG; $\$ 81$ Abs. 4 und $\S 84$ Abs. 2 SGB IX). Hier ließen sich aber auch die erwähnten Ansprüche auf Teilzeitarbeit einordnen.

Solche Gestaltungsansprüche stellen Anknüpfungspunkte für garantierte Optionen dar (Kocher 2007). Ihre Verwirklichung ist aber rechtlich nach wie vor davon abhängig, dass der Arbeitszeitwunsch der Beschäftigten in das Organisationskonzept des Arbeitgebers passt - auch hier ist der Anspruch also abhängig von Dispositionsmöglichkeiten der Unternehmen, wodurch die Interessendurchsetzung letztlich der Verhandlung mit dem Arbeitgeber überantwortet wird (ebd.). Auch scheint die biografische Option, das Recht auf Teilzeitarbeit mit den sozialversicherungsrechtlichen Langzeitkonten zusammen als effektives Recht auszugestalten, bislang nicht genutzt zu werden.

\subsection{RECHTE GESUNDHEITLICH EINGESCHRÄNKTER ERWERBS- PERSONEN}

Im Schwerbehinderten- und Rehabilitationsrecht sind gezielt aufeinander aufbauende arbeits- und sozialrechtliche Regelungen geschaffen worden, die verhindern sollen, dass Menschen wegen gesundheitlicher Einschränkungen aus dem Erwerbsleben ausscheiden müssen. Diese beinhalten auch die Möglichkeit, durch stufenweise Wiedereingliederung, Arbeitsplatzanpassung und betriebliches Eingliederungsmanagement Übergänge in eine dem Gesundheitszustand angemessene Form der Beschäftigung zu finden und zu fördern ( $\$ \$ 28,81,84$ SGB IX). Auf die Anpassung von Arbeitsbedingungen und die kooperative Gestaltung von Übergängen besteht ein Rechtsanspruch (BAGE 118, 252, BSG, SozR 4-3250 \$28 Ziff. 3; Kohte 2008; Nebe 2008; Welti 2008a).

Mit ihrem Ineinandergreifen arbeits- und sozialrechtlicher Instrumente könnten die Rechte gesundheitlich eingeschränkter Erwerbspersonen interessante und innovative Vorbilder auch für andere Übergangssituationen darstellen. Das normative Vorbild des Behindertenrechts zeigt insbesondere, wie arbeits- und sozialrechtliche Regelungsinstrumente komplementär wirken können. Dies ist für die
Risikosicherung in Übergangssituationen wohl unabdingbar: Arbeitsrechtliche Instrumente verteilen Risiken zwischen einzelnen Unternehmen und ihren Beschäftigten, während sozialrechtliche Instrumente die Finanzierungsverantwortung gesellschaftlich verteilen. In Übergangssituationen geht es darum, die Finanzierungs- und Gewährleistungsverantwortung zwischen Unternehmen, Beschäftigten und Sozialleistungsträgern angemessen zu verteilen - also um die Zusammenschau arbeitsrechtlicher und sozialrechtlicher Instrumente. Damit können Fragen der internen und externen Flexibilität gleichzeitig in den Blick genommen werden, denn beide Formen sind häufig funktional äquivalent und müssen als Optionen von Unternehmen sowie der Beschäftigten im Vergleich betrachtet werden.

Kooperation und Serviceleistungen der Sozialleistungsträger, vor allem der Übergang zwischen der Verantwortlichkeit verschiedener Sozialleistungsträger im Bereich der Rehabilitation sind allerdings noch sehr ausbaufähig (Welti 2008b). Selbst wo an sich lückenlose Regelungen bestehen, sind die Verwaltungspraxis und Beratungsqualität an Schnittstellen häufig defizitär und führen nicht selten zu unproduktiven Verzögerungen oder gar zum Verzicht auf mögliche Gestaltungen. So konnte das sinnvolle Instrument der stufenweisen Wiedereingliederung erkrankter Beschäftigter (\$28 SGB IX; Nebe 2008) bislang wegen Zuständigkeitsstreitigkeiten zwischen Kranken- und Rentenversicherung nicht voll genutzt werden. Gestaltungsmöglichkeiten durch freiwillige Versicherung sind oft nicht hinreichend bekannt.

Darüber hinaus zeigen die Erfahrungen mit der Arbeitsplatzanpassung bei gesundheitlichen Problemen auch, dass ihre Umsetzung vor allem in Klein- und Mittelbetrieben Probleme aufwirft, da diese es schwerer haben, aus eigener Kraft Arbeitsbedingungen anzupassen (Köpke 2009). Hier fehlt es noch an Modellen für die soziale Sicherung jenseits des Einzelunternehmens, z. B. durch überbetriebliche, insbesondere tarifliche Regelungsinstrumente, die Risiken überbetrieblich verteilen können. Von Interesse sind hier Vorbilder im Bereich der Urlaubskassen, der Zusatzversorgungskassen und der Qualifizierungstarifverträge.

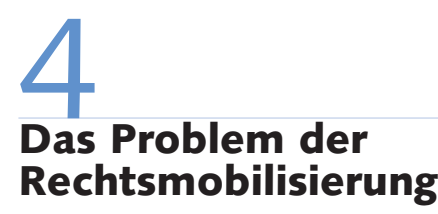

Selbst wenn, wie dargestellt, Instrumente für ein Soziales Recht der Arbeit zumindest punktuell vorhanden sind, bleiben sie häufig wirkungslos. Denn oft werden rechtliche Instrumente in der Praxis auch dann nicht durchgesetzt, wenn sie die Rechtsposition von Beschäftigten substanziell verbessern. Dieses Problem des Durchsetzungsdefizits ist schon seit Langem als Problem der „Rechtsmobilisierung“ erkannt (Cappelletti/Garth 1978; Blankenburg 1980), und es ist insbesondere in Konstellationen des Machtungleichgewichts wie im Arbeitsrecht relevant. Die bisher erlangten empirischen Erkenntnisse in der deutschen Arbeitsrechtssoziologie konzentrieren sich dabei auf die Frage, inwieweit der Kündigungsschutz effektiv durchgesetzt und mobilisiert wird (u.a. Pfarr et al. 2005). Jedoch: Solche Erkenntnisse können nicht einfach auf die Mobilisierung von Rechten auf Autonomie und sozialen Schutz in der Beschäftigung übertragen werden, denn die Zugangsbarrieren zum Rechtsschutz sind in der Situation des bestehenden Arbeitsverhältnisses andere als im Kündigungsschutz. Es ist zu vermuten, dass auch hier das Vertrauen auf die Initiative der betroffenen Beschäftigten bei der Mobilisierung ihrer Rechte nicht unbedingt gerechtfertigt ist. Gewiss ist das aber nicht, denn es fehlt an belastbaren Erkenntnissen über die effektive Nutzung solcher Rechte. Offen ist auch die Frage, wie die betriebliche Nutzung von Optionen im konkreten Einzelfall angestoßen werden kann, ohne die Betroffenen ausschließlich auf den Rechtsweg zu verweisen (genauer zum Forschungsbedarf Kocher 2009).

Auch im Sozialrecht werden bestehende Regelungen oft nicht genutzt. Dies kann Folge betrieblicher Machtverhältnisse sein. Es kann aber auch am Informations- und Machtgefälle liegen, das zwischen Bürgerinnen und Bürgern und Sozialleistungsträgern besteht. Dass jedenfalls die Sozialversicherungsträger durch eigene Normsetzung und durch die Mitwirkung der Gewerkschaften an der Selbstverwaltung näher bei den Beschäftigten sein sollten, ist heute allzu häufig nicht mehr zu spüren (Braun et al. 2008). So werden die Leistungsansprüche bei gesundheitlichen 
Einschränkungen oft erst dann eingefordert und anerkannt, wenn die Gesundheitsstörung weit fortgeschritten ist und eine endgültige Ausgliederung aus dem Arbeitsleben droht.

Innerbetrieblich wie außerbetrieblich ist die Realisierung von Rechten an Bera- tung und Unterstützung gebunden. Für Gewerkschaften und Arbeitgeber bedeutet dies ein großes, oft noch ungenutztes Handlungsfeld: Im Betrieb können Betriebsvereinbarungen und im sozialen $\mathrm{Si}$ cherungssystem können qualifizierte Beratungsstellen - wie z. B. die Servicestellen für
Rehabilitation - dazu beitragen, dass neue Regulierungsformen auch Realität werden. Wenn Arbeitsrecht und soziale Sicherheit näher bei den individuellen Lebensläufen sein sollen, werden auch betriebliche, tarifliche und satzungsmäßige Gestaltungskompetenzen gestärkt werden müssen.

\section{LITERATUR}

Berger-Delhey, U. (2009): "Certe ignoratio futurorum malorum utilior est quam scientia“ - Das Pflegezeitgesetz in der Praxis, in: Zeitschrift für Tarifrecht 3, S. 128-130

Bieback, K.-J. (1999): Neue Selbständigkeit und soziale Sicherheit Notwendigkeit einer Neuorientierung, in: Sozialer Fortschritt 7,

S. $166-174$

Biehl, S. (2004): Mindestkrankengeld für hauptberuflich selbständig Erwerbstätige - systemkonform oder ein Verstoß gegen die Entgeltersatzfunktion des Krankengeldes?, in: Die Sozialgerichtsbarkeit 11, S. 678-684

Blanke, T./Bleses, P. (2005): Flexicurity im Arbeits- und Sozialleistungsrecht in: Kronauer, M./Linne, G. (Hrsg.): Flexicurity. Die Suche nach Sicherheit in der Flexibilität, Berlin, S. 365-384

Blankenburg, E. (1980): Mobilisierung von Recht, in: Zeitschrift für Rechtssoziologie 1, S. 33-64

Bogedan, C. (2008): Pflegeverantwortung zwischen Familie und StaatEin deutsch-dänischer Vergleich, in: WSI-Mitteilungen 4, S. 212-218 Brandl, S./Hildebrandt, E./Wotschak P. (Hrsg.) (2008): Arbeitszeitpolitik im Lebenslauf - Ambivalenzen und Gestaltungsoptionen in deutscher und europäischer Perspektive, Düsseldorf

Braun, B./Klenk, T./Kluth, W./Nullmeier, F./ Welti, F. (2008): Modernisierung der sozialen Selbstverwaltung und der Wahl ihrer Vertreter, in: Soziale Sicherheit 8, S. 245-250

Brinkmann, U./Dörre, K./Röbenack, S. (2006): Prekäre Arbeit. Ursachen, Ausmaß, soziale Folgen und subjektive Verarbeitungsformen unsicherer Beschäftigungsverhältnisse, Bonn

Cappelletti, M./Garth, B. (Hrsg.) (1978): Access to Justice, Vol. I: A World Survey, Vol. II: Promising Institutions, Vol. III: Emerging Issues and Perspectives, Vol. IV: Anthropological Perspectives, Alphenaandenrijn/ Mailand

Döring, D. (2009): Versicherungspflicht in der gesetzlichen Rentenversicherung und Erwerbstätigkeit, in: Deutsche Rentenversicherung 3, S. 309-318

Europäische Kommission (2006): Grünbuch vom 22.11.2006 „Ein modernes Arbeitsrecht für die Herausforderungen des 21. Jahrhunderts“, $\operatorname{KOM}(2006) 708$ endg.

Hanau, H. (2005): Die „betrieblichen Gründe“ des § 8 Abs. 4 Satz 1 TzBfG im Lichte aktueller Entscheidungen des BAG, in: Recht der Arbeit 5, S. 301-305.

Hanau, P. (2005): Arbeitsrechtliche Rahmenbedingungen flexibler Beschäftigung, in: Kronauer, M./Linne, G. (Hrsg.): Flexicurity. Die Suche nach Sicherheit in der Flexibilität, Berlin, S. 347-364

Hanau, P./Veit, A. (2009): Neues Gesetz zur Verbesserung der Rahmenbedingungen für die Absicherung flexibler Arbeitszeitregelungen und zur Änderung anderer Gesetze, in: Neue Juristische Wochenschrift 4, S.182-187

Hildebrandt, E. (Hrsg) (2007): Lebenslaufpolitik im Betrieb. Optionen zur Gestaltung der Lebensarbeitszeit durch Langzeitkonten, Berlin Keller, B./Seifert, H. (2007): Atypische Beschäftigungsverhältnisse: Flexibilität, soziale Sicherheit und Prekarität, in: Keller, B./Seifert, H.: Atypische Beschäftigung - Flexibilisierung und soziale Risiken, Berlin, S. $11-25$

Keller, B./Seifert, H. (2008): Flexicurity: Ein europäisches Konzept und seine nationale Umsetzung, Expertise für die Friedrich-Ebert-Stiftung, Bonn

Kerschbaumer, J. (2009): Flexibler Ausstieg aus dem Erwerbsleben aber wie?, in: Soziale Sicherheit 4, S. 125-132

Klammer, U. (2005): Flexicurity aus der Perspektive des Lebenslaufs, in: Kronauer, M./ Linne, G.(Hrsg.): Flexicurity. Die Suche nach Sicherheit in der Flexibilität, Berlin, S. 249-273

Klenner, C./Pfahl, S. (2008): Jenseits von Zeitnot und Karriereverzicht - Wege aus dem Arbeitszeitdilemma. Analyse der Arbeitszeiten von Müttern, Vätern und Pflegenden und Umrisse eines Konzeptes, WSIDiskussionspapier 158, Düsseldorf

Kocher, E. (2007): Gestaltungs- und Verhandlungsrechte von Beschäftigten, in: WSI-Mitteilungen 8, S. 434-440

Kocher, E. (2009): Effektive Mobilisierung von Beschäftigtenrechten. Das Arbeitsrecht in der betrieblichen Praxis, Reihe Böckler Forschungsmonitoring, Düsseldorf

Kohte, W. (2008): Betriebliches Eingliederungsmanagement und Bestandsschutz, in: Der Betrieb 11, S. 582-587

Köpke, K.-H. (2009): Praktische Erfahrungen mit dem betrieblichen Eingliederungsmanagement, in: Soziale Sicherheit 2, S. 68-73

Mückenberger, U. (2007): Ziehungsrechte - Ein zeitpolitischer Weg zur "Freiheit in der Arbeit“, in: WSI-Mitteilungen 4, S. 195-201

Nebe, K. (2008): (Re-)Integration von Arbeitnehmern: Stufenweise Wiedereingliederung und Betriebliches Eingliederungsmanagement ein neues Kooperationsverhältnis, in: Der Betrieb 33, S.1801-1805 Nickel, H. M. (1999): Industriegesellschaft am Ende - Arbeit abgeschafft? - Frauen und der Geschlechterkampf um Erwerbsarbeit, in: Stolz-Willig, B./Veil, M. (Hrsg.): Es rettet uns kein höheres Wesen Feministische Perspektiven der Arbeitsgesellschaft, Hamburg, S. 9-28 Opitz, H. C. (2005): Der juristische und gesellschaftliche Umgang mit den Ansprüchen auf Teilzeitarbeit in den Niederlanden und in Deutschland, in: Kritische Justiz 2, S. 164-177

Ostner, I. (1995): Arm ohne Ehemann? Sozialpolitische Regulierung von Lebenschancen für Frauen im internationalen Vergleich, in: Aus Politik und Zeitgeschichte 36/37, S. 3-12 
Pfarr, H. (2000): Soziale Sicherheit und Flexibilität: Brauchen wir ein

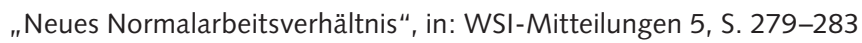
Pfarr, H./Ullmann, K./Bradtke, M./Schneider, J./Kimmich, M./Bothfeld, S. (2005): Der Kündigungsschutz zwischen Wahrnehmung und Wirklichkeit. Betriebliche Erfahrungen mit der Beendigung von Arbeitsverhältnissen, München

Radbruch, G. (1999): Rechtsphilosophie, Heidelberg

Reinhard, H.-J. (2009): Modelle der Erwerbstätigensicherung in Europa, in: Deutsche Rentenversicherung 3, S. 319-328

Rolfs, C./Witschen, S. (2009): Neue Regeln für Wertguthaben,

in: Neue Zeitschrift für Sozialrecht 6, S. 295-302

Schietinger, M. (2008): Plädoyer für eine solidarische und umlagefinanzierte Lösung - Insolvenzsicherung von Arbeitszeitguthaben, in: Soziale Sicherheit 11 , S. 380-385

Schmid, G. (2002): Wege in eine neue Vollbeschäftigung. Übergangsarbeitsmärkte und aktivierende Arbeitsmarktpolitik, Frankfurt/New York Sennett, R. (2000): Der flexible Mensch. Die Kultur des neuen Kapitalismus, Berlin

Stolz-Willig, B. (2001): In welcher Gesellschaft leben wir eigentlich? Über die Abwesenheit einer geschlechterdemokratischen Perspektive in der Debatte zur Zukunft der Arbeit in: Stolz-Willig, B. (Hrsg.): Arbeit und Demokratie, Hamburg, S. 52-66

Supiot, A. (2001): Beyond Employment: Changes at Work and the Future of Employment Law in Europe, Oxford

Supiot, A./Casas, M.-E./Hanau, P./Johansson, A./Meadows, P./ Mingione, E./Salais, R./van der Heijden, P. (1999): A European Per- spective on the Transformation of Work and the Future of Labour Law, in: Comparative Labour Law \& Policy Journal 20, S. 621-633

Voß, G.G./Pongratz, H.J. (1998): Der Arbeitskraftunternehmer - Eine neue Grundform der Ware Arbeitskraft?, in: Kölner Zeitschrift für Soziologie und Sozialpsychologie 1, S. 131-158

Waas, B. (2000): Gesetzlicher Anspruch auf Teilzeitarbeit in den Niederlanden, in: Neue Zeitschrift für Arbeitsrecht 10, S. 583-585

Waddington, L./Hendriks, A. (2002): The expanding Concept of Employment Discrimination in Europe: From Direct and Indirect Discrimination to reasonable Accommodation Discrimination, in: International Journal of Comparative Labour Law and Industrial Relations 4,

S. 403-428

Welti, F. (2001a): Wandel der Arbeit und Reform von Sozialstaat und Sozialrecht, in: Sozialer Fortschritt 3, S. 69-79

Welti, F. (2001b): Sozialversicherung für Selbständige öffnen - Bedürfnis nach sozialem Schutz wächst, in: Soziale Sicherheit 6-7, S. 223-229 Welti, F. (2008a): Betriebliches Eingliederungsmanagement: Die Aufgaben und Pflichten der Sozialleistungsträger, in: Soziale Sicherheit 4, S. $125-129$

Welti, F. (2008b): Systematische Stellung des SGB IX im Sozialgesetzbuch - Zusammenarbeit der Leistungsträger und Koordination der Leistungen, in: Die Sozialgerichtsbarkeit 6, S. 321-331

Wotschak, P./Hildebrandt, E./Scheier, F. (2008): Langzeitkonten Neue Chancen für die Gestaltung von Arbeitszeiten und Lebensläufen?, in: WSI-Mitteilungen 11+12, S. 619-626. 